\title{
Una propuesta para introducir la enseñanza de las Pruebas de Hipótesis
}

\author{
Giovanni Sanabria Brenes. \\ gsanabria@itcr.ac.cr, \\ Instituto Tecnológico de Costa Rica - Universidad de Costa Rica
}

\section{Resumen.}

El presente trabajo brinda una propuesta que busca enfrentar el problema de enseñanza de los conceptos asociados a las pruebas de hipótesis. La propuesta plantea varias situaciones problema en forma de ejemplos que permiten reforzar la comprensión de ciertos conceptos asociados a las pruebas de hipótesis.

Palabras clave: Didáctica, Pruebas de hipótesis, Decisión, Errores.

\section{Abstract.}

This paper provides a proposal that seeks to approach the problem of teaching the concepts associated with hypothesis testing. The proposal raises several problem situations, in examples way, to reinforce understanding of certain concepts related to hypothesis testing.

Keywords: Teaching, hypothesis testing, decision, errors.

\subsection{Introducción}

El contraste de pruebas de hipótesis es una herramienta muy importante a utilizar en las investigaciones cuantitivas para la toma de decisiones. Precisamante su fortaleza está en ayudarnos a tomar las decisiones que no necesariamente son las correctas pero si aquellas que tiene una mayor probabilidad de ser las correctas. Además, el constraste de hipótesis tiene aplicaciones en diversas áreas del conocimiento lo cual contribuye a su importancia.

Sin embargo, la enseñanza de las pruebas de hipótesis presentan varias dificultades, tres de estás dificultades son detectadas por Vallecillos \& Batanero (1997) a partir de un estudio de caso:

1. Involucra un gran número de conceptos que se asume su dominio por parte del estudiante: nivel de significacia, hipótesis nula, hipótesis alternativa, valor $\mathrm{P}, \ldots$

2. No establece relaciones entre los conceptos, por ejemplo no establecen relación entre la distribución del estadístico, las regiones y el nivel de significación 
3. Los estudiantes pueden dar respuestas a los problemnas planteados sin conectar los conceptos.

El presente trabajo brinda una propuesta para introducir las pruebas de hipótesis que pretender superar las dificultades anteriores. Esta propuesta ha sido plasmada en texto que se ha utilizado en el Instituto Tecnológico de Costa Rica en los últimos semestres para el curso $M A-3405$ Estadística y los resultados han sido satisfactorios.

\subsection{Concepto intuitivo}

Para introducir el concepto de pruebas de hipótesis se tomará como base la propuesta desarrollada por Martha Aliaga (2008). Suponga que se tiene una bolsa con 6 bolas de las cuales no se conoce su color:

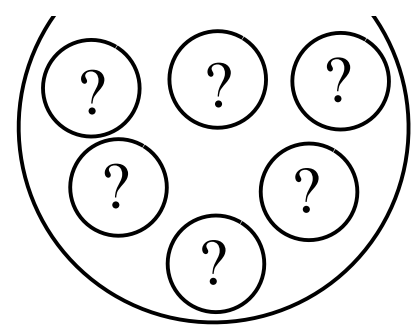

Una persona realiza la siguiente afirmación: "en la bolsa hay 5 bolas verdes y una blanca" y le solicitan a usted que rechace o acepte la afirmación, para ello le dan la posibilidad de realizar una muestra aleatoria de tamaño 2. Es decir le permiten elegir dos bolas al azar con reposición de la bolsa.

Suponga que al realizar las dos extracciones con reposición obtiene dos bolas blancas:

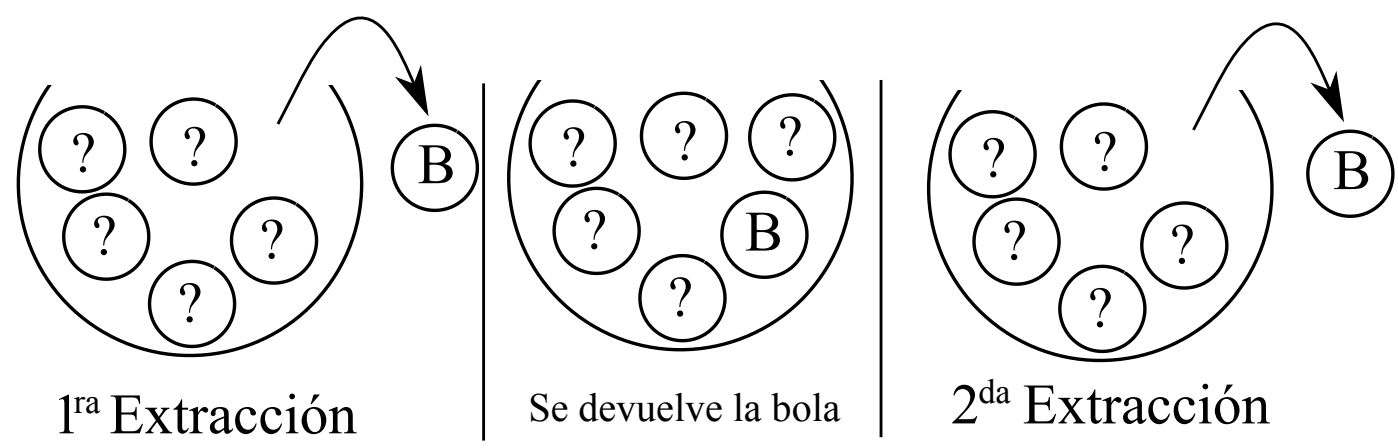

Con base en la información obtenida en la muestra, responda las siguientes preguntas:

1. ¿Acepta o rechaza la afirmación de la persona?

2. ¿Hay alguna posibilidad de que se cumpla la afirmación?

3. ¿Considera más probable que la afirmación se correcta o incorrecta?

4. Discuta la diferencia, con respecto a la afirmación, entre la toma de una decisión (aceptar o rechazar la afirmación) y la asignación de un valor de verdad a la afirmación (afirmación correcta o incorrecta).

Suponga que la bolsa fue desechada y nunca se determinó la veracidad de la afirmación, entonces ¿puede asegurar que su decisión fue la correcta o la más probable?. 
Cuando se toman decisiones con respecto a la información brindada por una muestra se realiza una prueba de hipótesis. Discuta la diferencia entre una prueba de hipótesis y una prueba matemática.

\subsection{Conceptos básicos}

\subsubsection{Tipos de hipótesis}

La investigación define las hipótesis como las afirmaciones que son objeto de estudio y que se desea determinar su veracidad. La prueba de hipótesis es un método de investigación que a partir de una afirmación, sobre el valor de un parámetro, define dos hipótesis opuestas (una de las cuales es la afirmación) y busca evidencias para aceptarlas o rechazarlas. Seguidamente se recuerdan las definiciones de los dos tipos de hipótesis.

Definición 1.1 (Hipótesis nula). (Acusado es inocente). Es una aseveración en el sentido de que un parámetro $\theta$ tenga un valor específico $\theta_{0}$ y se denota $H_{0}$ :

$$
H_{0}: \theta=\theta_{0}
$$

donde $\theta_{0}$ es un valor del estadístico $\widehat{\Theta}$ llamado valor nulo. El procedimiento consiste en suponer que $H_{0}$ es verdadera y buscar evidencias en contra del supuesto. Para que $H_{0}$ se acepte no debe haber una fuerte evidencia en su contra.

Definición 1.2 (Hipótesis alternativa). (Acusado es culpable). es una aseveración que se acepta si se rechaza $H_{0}$, se denota por $H_{1}$ y tiene la forma:

$$
H_{1}: \theta<\theta_{0}, \quad H_{1}: \theta>\theta_{0} \quad o \quad H_{1}: \theta \neq \theta_{0}
$$

Observaciones: Se tiene que

1. De acuerdo con la ley de tricotomía:

$$
\theta=\theta_{0}, \quad \theta<\theta_{0} \quad o \quad \theta>\theta_{0}
$$

$H_{0}$ y $H_{1}$ deben abordar estas afirmaciones y ser opuestas.

2. Aunque $H_{0}$ se redacta en forma de igualdad, en realidad suele ser su caso extremo para que se cumpla. Por ejemplo, si se desea estudiar la afirmación $\theta \leq \theta_{0}$, entonces, dado que la afirmación debe estar sola en una de las hipótesis para poder analizarla, se tiene que

$$
H_{0}: \theta=\theta_{0} \quad(\leq) \quad H_{1}: \theta>\theta_{0}
$$

Observe que la afirmación, dado que contiene la igualdad $\left(\theta \leq \theta_{0}\right)$, se le asigna a $H_{0}$. Así, $H_{0}$ se acepta si $\theta \leq \theta_{0}$ y su caso extremo para que se acepte es que $\theta=\theta_{0}$. Por otro lado, en $H_{1}$ se le asigna lo contrario a la afirmación.

3. Recuerde que "el acusado es inocente hasta que se demuestre lo contrario". Por lo tanto, hasta el momento el procedimiento para realizar una prueba de hipótesis consiste en:

(a) Definir $H_{0}$ y $H_{1}$ a partir de la afirmación por analizar 
(b) Asumir $H_{0}$ es verdadero, es decir se asume que "el acusado es inocente".

(c) Tomar una muestra para hallar un valor $\widehat{\theta}$ del estadístico $\widehat{\Theta}$

(d) Utilizar la estimación $\widehat{\theta}$ y la distribución de $\widehat{\Theta}$ para determinar evidencias en contra de $H_{0}$, es decir "se buscan evidencias que se demuestre lo contrario".

(e) Decisión. Si se determinar evidencias significativas en contra de $H_{0}$, entonces se rechaza $H_{0}$. De lo contrario se acepta $H_{0}$.

(f) Conclusión. Si se acepta $H_{0}$, no significa que se acepta que "el acusado es inocente" sino, al igual que en un juicio, se concluye que no se hallaron evidencias significativas en contra de $H_{0}$. Por otro lado si se rechaza $H_{0}$, se concluye que existe evidencia en contra de $H_{0}$.

Ejemplo 1.1 Para cada una de las siguientes situaciones identifique el parámetro, indique la afirmación a analizar y, con base en esta, identifique la hipótesis nula y alternativa.

1. Se quiere demostrar que el nivel promedio de carbono del centro de San José es mayor que 4.9 partes por millón.

Sea $\mu$ el nivel promedio de carbono del centro de San José.

$$
\begin{gathered}
\text { Afirmación }: \mu>4.9 \\
H_{0}: \mu=4.9 \quad(\leq) \quad H_{1}: \mu>4.9
\end{gathered}
$$

2. Se desea determinar si el porcentaje de costarricenses que duermen con el televisor encendido es menor a $35 \%$.

Sea $p$ el porcentaje de costarricenses que duermen con el televisor encendido.

$$
\begin{gathered}
\text { Afirmación }: p<0.35 \\
H_{0}: p=0.35 \quad(\geq) \quad H_{1}: p<0.35
\end{gathered}
$$

Definición 1.3 Una prueba de una cola es aquella en la cual $H_{1}$ tiene la forma:

$$
H_{1}: \theta<\theta_{0} \quad o \quad H_{1}: \theta>\theta_{0}
$$

Definición 1.4 Una prueba de dos colas es aquella en la cual $H_{1}$ tiene la forma:

$$
H_{1}: \theta \neq \theta_{0}
$$

\subsubsection{Regiones de aceptación y rechazo}

La aceptación de $H_{0}$ se da si al tomar una muestra no se encuentra evidencia significativa en su contra. Es decir la aceptación de $H_{0}$ no está directamente ligada a la posibilidad de que se cumpla $H_{0}$ (aunque si indirectamente), más bien su aceptación se da cuando no existan datos en su contra. Por lo tanto, el concepto de aceptación en pruebas de hipótesis es distinto al de la vida cotidiana.

Ejemplo 1.2 Juan afirma que Ana tiene puesta una camisa de color rosada. Se tiene que

$H_{0}: \quad$ la camisa que tiene puesta Ana es rosada

$H_{1}$ : la camisa que tiene puesta Ana no es rosada 
Se entrevistan a cuatro personas que vieron Ana: Luis indica que la camisa de Ana no es de color verde, Rebeca dice que no es roja, Alberto que no recuerda el color y Sara indica que no es amarrilla. Por lo tanto, dado que la información obtenida no brinda evidencia en contra de $H_{0}$, se acepta que la camisa es rosada, esto pese a que directamente no se obtuvo información de que fuera de dicho color. Así,

$$
\text { se acepta } H_{0}
$$

y lo correcto es concluir que no se hallan evidencias en contra de que sea rosada

Ejemplo 1.3 Considere el ejemplo anterior. Suponga que Anthony afirma que Ana tiene puesta una camisa de color negra. Así,

$$
\begin{aligned}
& H_{0}: \quad \text { la camisa que tiene puesta Ana es negra } \\
& H_{1}: \text { la camisa que tiene puesta Ana no es negra }
\end{aligned}
$$

De acuerdo a la información indicada en el ejemplo anterior, no hay evidencia significativa para rechazar $H_{0}$. Por lo tanto,

$$
\text { se acepta que la camisa es negra }
$$

Sin embargo, en el ejemplo anterior, se obtuvo que

$$
\text { se acepta que la camisa es rosada }
$$

¿Se contradicen (1) y (2)? No, en realidad se acepta que la camisa es negra y que es rosada, al no existir evidencia en contra de ambas afirmaciones. Es decir, la aceptación se da porque no se halló un argumento sólido para negarla.

Los ejemplos anteriores modelan el concepto de aceptación en una prueba de hipótesis e indicar que es posible aceptar dos afirmaciones contradictorias si no evidencia significativa para rechazarlas.

¿Cómo aceptar o rechazar $H_{0}$ ? La hipótesis nula realiza una afirmación sobre el valor de un parámetro $\theta$. Recuerde que $\widehat{\Theta}$, un buen estimador o estadístico asociado a $\theta$, brinda estimaciones sobre el valor $\theta$ a partir de muestras de tamaño fijo. Así, la idea es utilizar una estimación $\widehat{\theta}$ de $\theta$ y la distribución muestral de $\widehat{\Theta}$ para determinar evidencia en contra de $H_{0}$. Por lo tanto, el valor de la estimación de $\theta$ es trascendental para aceptar o rechazar $H_{0}$.

Ejemplo 1.4 Se afirma que el promedio de edad de los vendedores del Mercado Central de Cartago es de por lo menos 30 años.

Para analizar esta afirmación, sea $\mu$ la edad promedio de los vendedores del Mercado Central de Cartago. Así,

$$
\begin{gathered}
\text { afirmación }: \mu \geq 30 \\
H_{0}: \mu=30 \quad(\geq) \quad H_{1}: \mu<30
\end{gathered}
$$

El estadístico utilizado es $\bar{X}$. Recuerde que este estadístico brinda estimaciones de $\mu$ para muestras de tamaño 40, por ejemplo.

1. Suponga que en una muestra de tamaño 40 se halló el valor $\bar{x}=32$. Como $\bar{x}$ es una estimación de $\mu$, esto indica que $\mu$ es cercano a 32, y recuerde que $H_{0}$ se cumple si $\mu \geq 30$. Así, el valor $\bar{x}=32$ no representa una evidencia en contra de $H_{0}$, por lo tanto

$$
\text { se acepta } H_{0} \text {. }
$$

2. Suponga que en una muestra de tamaño 40 se halló el valor $\bar{x}=29$.Esto indica que $\mu$ es cercano a 29. Así, se ha determinado evidencia en contra de $H_{0}$. Sin embargo, ¿esta evidencia es significativa?

(a) Recuerde que $\bar{x}=29$ es solo una estimación de $\mu$. Se sabe que $\mu$ es cercano a 29 , entonces $\mu$ puede ser 30 el cual se supone cercano a 29. Por lo tanto, se puede considerar que la evidencia no es significativa y

$$
\text { se acepta } H_{0} \text {. }
$$


(b) Por otro lado, se puede considerar que 30 está alejando de 29. Así, la evidencia es significativa y

$$
\text { se rechaza } H_{0} \text {. }
$$

(c) ¿29 es cercano o no a 30? Esto dependerá de la distribución del estadístico $\bar{X}$ y del riesgo de equivocarse que se está dispuesto a asumir. Como se verá más adelante, si se considera que como $\bar{x}=29.9$ entonces $\mu<30$ (29.9 está alejado de 30) entonces se está aumentando el riesgo de cometer un error. En general, si estimaciones muy cercanas a 30 pero menores se consideran significativas para rechazar $H_{0}$, se aumenta el riesgo de equivocarse en la decisión tomada.

Continuando con la propuesta, se definen las regiones de una prueba de hipótesis.

Definición 1.5 Dado que

$$
H_{0}: \theta=\theta_{0}
$$

se define

a.) Región de aceptación: Valores de $\widehat{\Theta}$, estimaciones de $\theta$, que aceptan $H_{0}$

b.) Región de rechazo: Valores de $\widehat{\Theta}$, estimaciones de $\theta$, que rechazan $H_{0}$

c.) Valor crítico: separa una región de rechazo y una de aceptación. Se denota $\theta_{c}$.

Aquí es importante que el docente presente las regiones de aceptación y rechazo para cada prueba de hipótesis:

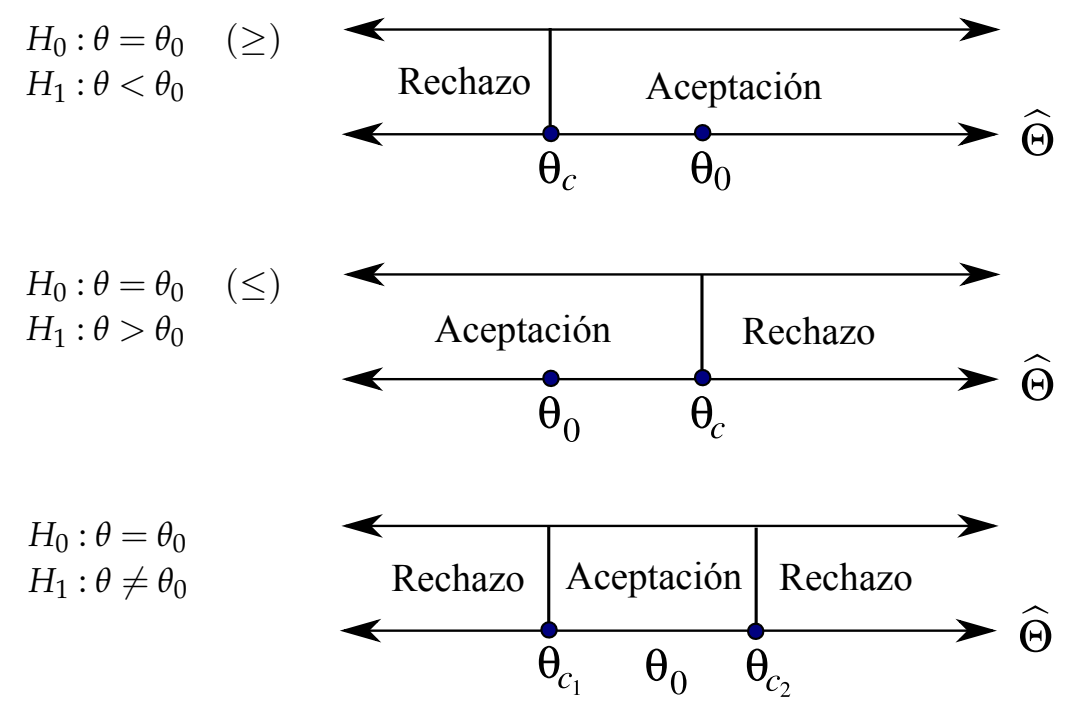

El lector puede notar que hay una tolerancia en cada una de las pruebas para considerar que $\theta=\theta_{0}$, tolerancia ejemplificada en el ejemplo anterior. De manera general, considere la primera prueba donde $H_{0}$ es

$$
H_{0}: \theta=\theta_{0} \quad(\geq)
$$

Entonces, para valores del estadístico (estimador de $\theta$ ) muy cercanos a $\theta_{0}$, aunque menores a este valor nulo, se considera que $\theta=\theta_{0}$ y por lo tanto debe formar parte de la región de aceptación. 
Esta tolerancia, que diferencia el valor crítico $\theta_{c}$ y el valor nulo $\theta_{0}$, se justifica debido a la utilización del estadístico $\widehat{\Theta}$ cuyos valores son estimaciones de $\theta$ tomadas de una muestra. Así, si las estimaciones de $\theta$ son muy cercanas a $\theta_{0}$ se puede aceptar que $\theta=\theta_{0}$.

La distancia entre el valor nulo y el valor crítico dependerá del riesgo que se este dispuesto a tomar al realizar la prueba de hipótesis. Entre más lejanos están estos valores, se considera que estimaciones cercanas a $\theta_{0}$ aceptan $H_{0}$ y hay menos posibilidad de cometer el error de rechazar $H_{0}$ siendo verdadera. Esto se estudiará en el siguiente apartado.

Así, la obtención del valor crítico dependerá de la distribución del estadístico y del riesgo que se está dispuesto a asumir al rechazar $H_{0}$ siendo verdadera. Esto se verá en el apartado (1.4).

Ejemplo 1.5 Se desea determinar si la proporción $p$ de apartamentos de San José que prohíben mascotas no es superior al 30\%.

Se tiene que

$$
\begin{gathered}
\text { Afirmación }: p \leq 0.3 \\
H_{0}: p=0.3 \quad(\leq) \quad H_{1}: p>0.3
\end{gathered}
$$

El estadístico a utilizar es $\widehat{P}$. Las regiones de aceptación y rechazo son

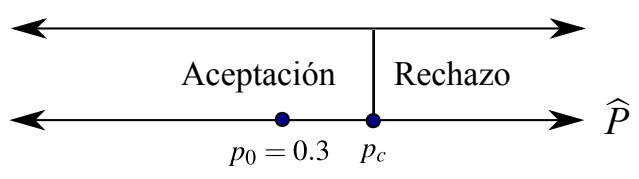

donde $p_{c}$ es el valor crítico.

\subsubsection{Errores en una prueba de hipótesis}

Para hablar de los errores de una prueba de hipótesis es común retomar la analogía de estas pruebas con un jucio. Al dictar sentencia en un juicio se pueden cometer dos tipos de errores: declarar culpable al acusado y que sea inocente, declarar inocente al acusado y que sea culpable Estos dos errores son un modelo para explicar los error que se pueden cometer cuando se ha realizado una prueba de hipótesis.

Definición 1.6 (Errores de una prueba de hipótesis) Se pueden presentar los siguientes errores:

a.) Error tipo I ("Condenar a un inocente") la hipótesis nula ("el acusado es inocente") se rechaza siendo verdadera. La probabilidad del error tipo I se denota por $\alpha$ y es la probabilidad de rechazar $H_{0}$ ("se declara culpable al acusado") sabiendo que $H_{0}$ es verdadera ("sabiendo el acusado es inocente"):

$$
\alpha=P\left(H_{1} \mid H_{0}\right)
$$

b.) Error tipo II ("Dejar libre al culpable") la hipótesis nula se acepta ("el acusado es inocente") siendo falsa. La probabilidad del error tipo II se denota por $\beta$ y es la probabilidad de aceptar $H_{0}$ ("se declara inocente al acusado") sabiendo que $H_{1}$ es verdadera ("sabiendo que el acusado es culpable"):

$$
\beta=P\left(H_{0} \mid H_{1}\right)
$$

Analicemos la probabilidad del error tipo I ("Condenar a un inocente"):

$$
\alpha=P\left(H_{1} \mid H_{0}\right)
$$


Note que $H_{1}$ se acepta (que es equivalente a rechazar $H_{0}$ ) para estimaciones de $\theta$ dadas por el estadístico $\widehat{\Theta}$ que se encuentran en la región de rechazo. Por otra lado dado que $H_{0}$ es verdadera, el caso extremo para que $H_{0}$ sea verdadero es que $\theta=\theta_{0}$. Así

$$
\alpha=P\left(H_{1} \mid H_{0}\right)=P\left(\widehat{\Theta} \text { este en la región de rechazo } \mid \theta=\theta_{0}\right)
$$

En cuando a la probabilidad del error tipo II ("Dejar libre al culpable”):

$$
\beta=P\left(H_{0} \mid H_{1}\right)
$$

Se tiene que $H_{0}$ se acepta para estimaciones de $\theta$, dadas por el estadístico $\widehat{\Theta}$, que se encuentran en la región de aceptación. Por otro lado, la probabilidad de este tipo de error solo podrá ser analizada para hipótesis alternativas específicas: $H_{1}^{\prime}: \theta=\theta_{1}$, donde $\theta_{1}$ es un valor específico de $\theta$ para el cual $H_{1}$ se cumple. Así la probabilidad del error tipo II para la hipótesis alternativa específica: $H_{1}^{\prime}: \theta=\theta_{1}$ es

$$
\beta=P\left(H_{0} \mid H_{1}^{\prime}\right)=P\left(\widehat{\Theta} \text { este en la región de aceptación } \mid \theta=\theta_{1}\right)
$$

Ejemplo 1.6 Suponga que la edad promedio $\mu$ de los niños de una cierta comunidad es mayor a 11, y suponga que la hipótesis alternativa específica es $H_{1}^{\prime}: u=12$. Redacte las hipótesis nula y alternativa, identifique el estadístico a utilizar y las regiones de aceptación y rechazo. Además redacte las probabilidades del error tipo I y del error tipo II para la hipótesis alternativa específica dada.

La afirmación es

$$
\text { afirmación : } \mu>11
$$

Recuerde que el estadístico asociado a $\mu$ es $\bar{X}$. Así se tiene que

$$
\begin{aligned}
& H_{0}: \mu=11 \quad(\leq) \\
& H_{1}: \mu>11
\end{aligned}
$$

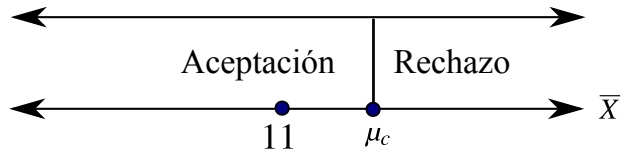

donde las probabilidades de los errores son

$$
\begin{aligned}
& \alpha=P\left(H_{1} \mid H_{0}\right)=P\left(\bar{X}>\mu_{c} \mid \mu=11\right) \\
& \beta=P\left(H_{0} \mid H_{1}^{\prime}\right)=P\left(\bar{X}<\mu_{c} \mid \mu=12\right)
\end{aligned}
$$

El ejemplo siguiente da evidencia de que $\alpha$ es la medida de la región de rechazo cuando se asume que $H_{0}$ es verdadera.

Ejemplo 1.7 Considere la prueba

$$
H_{0}: \theta=\theta_{0} \quad(\geq) \quad H_{1}: \theta<\theta_{0}
$$

En este caso, la probabilidad del error tipo I es

$$
\alpha=P\left(H_{1} \mid H_{0}\right)=P\left(\widehat{\Theta}<\theta_{\mathcal{c}} \mid \theta=\theta_{0}\right)
$$

Así, $\alpha$ es el área acumulada bajo la distribución de $\widehat{\Theta}$ hasta el valor crítico si se supone que $\theta=\theta_{0}$ :

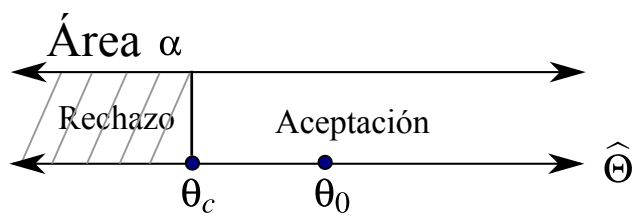

Por simplicidad se bosqueja la distribución de $\widehat{\Theta}$ como una línea horizontal. Sin embargo, el lector debe ser consciente de este hecho, por ejemplo si $\widehat{\Theta}$ es normal entonces realmente se tiene que 


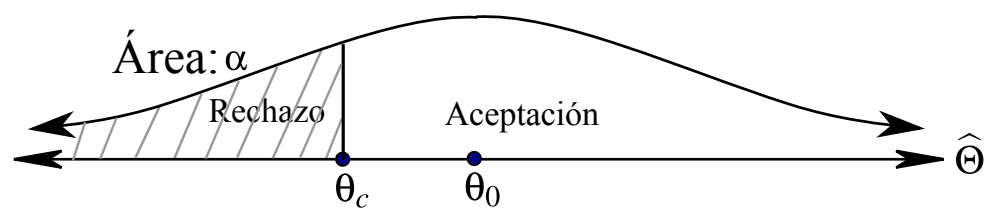

Teorema 1.1 La probabilidad del error Tipo I es la medida de la región o regiones de rechazo, asumiendo la hipótesis nula.

El error tipo I es mayor cuando el valor crítico $\theta_{c}$ es cercano al valor nulo $\theta_{0}$, pues aumenta la posibilidad de que estimaciones de $\theta$ cercanas a $\theta_{0}$ sean consideradas significativas para rechazar $H_{0}$, corriendo el riesgo de que $H_{0}$ se verdadera. Véase esto en el siguiente ejemplo.

Ejemplo 1.8 Se quiere concluir que la varianza $\sigma^{2}$ en la duración de las llantas Fire es igual a $1000 \mathrm{~km}^{2}$.

En este caso, se tiene que la afirmación es

$$
\text { afirmación : } \sigma^{2}=1000
$$

Recuerde que el estadístico asociado a $\sigma^{2}$ es $S^{2}$. Así se tiene que

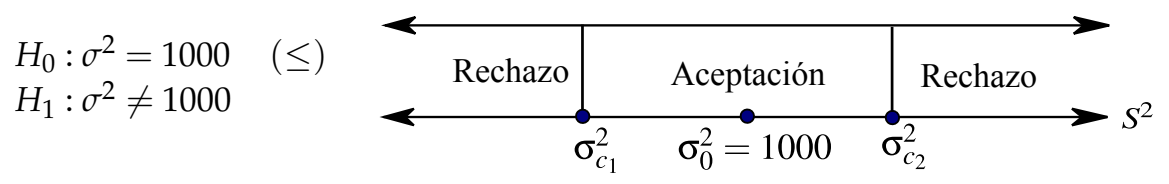

Suponga que en una muestra se determinó una estimación de $\sigma^{2}: s^{2}=1005$. ¿Es esta estimación significativa para rechazar $H_{0}$ ? Veamos las siguientes opciones:

1. Si los valores críticos están alejados del valor nulo $\sigma_{0}^{2}$. Suponga que por algún método, que se verá más adelante, se determinan los valores críticos $\sigma_{c 1}^{2}=990$ y $\sigma_{c 2}^{2}=1010$ :

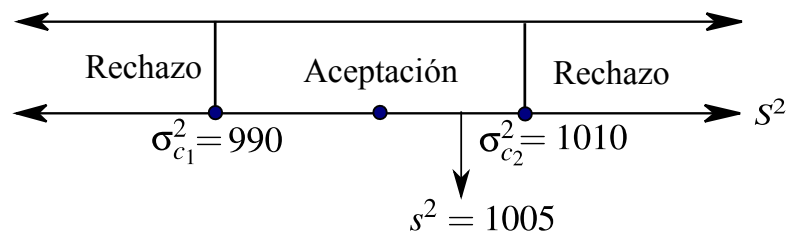

Dado que $s^{2}$ está en la región de aceptación, entonces no se considera significativo para rechazar $H_{0}$. Por lo tanto se acepta $H_{0}$.

2. Si los valores críticos están cercanos del valor nulo $\sigma_{0}^{2}$. Suponga que los valores críticos son $\sigma_{c 1}^{2}=996 \mathrm{y}$ $\sigma_{c 2}^{2}=1004$ :

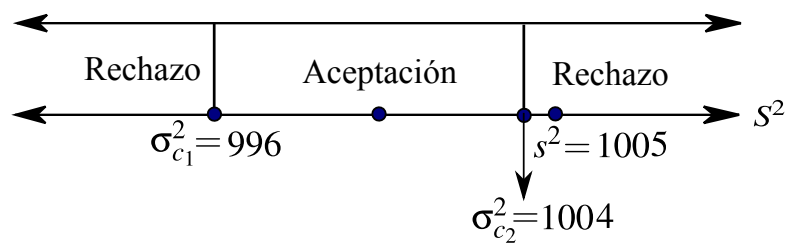

Dado que $s^{2}$ está en la región de rechazo, entonces se considera evidencia significativa en contra de $H_{0}$. Por lo tanto se rechaza $H_{0}$.

Comparando estas opciones, se puede notar que el error tipo I es mayor en (2) esto pues: 
(a) En (2) aumenta la probabilidad de que estimaciones como 1005 y 1004.1 sean consideradas significativas para rechazar $H_{0}$, esto no ocurre en (1). Así, en (2) hay más posibilidad de rechazar $H_{0}$ y por lo tanto de cometer el error tipo I.

(b) Utilizando el teorema anterior, las regiones de rechazo son mayor en (2) que en (1), por lo tanto el error tipo I es mayor en (2).

En cierta medida los errores son opuestos, si se tratada de evitar el error tipo I ("Condenar a un inocente") se puede caer en el error tipo II ("Dejar libre al culpable"). Esto hace que, solo se pueda controlar uno de los errores, el más grave.

¿Cuál de los errores es más grave? En un juicio se prefiere dejar libre a un culpable que condenar un inocente, es decir se considera más grave condenar a un inocente. Recuerde que "el acusado es inocente hasta que se demuestre lo contrario", entonces se controla el error tipo I, se acepta inicialmente $H_{0}$ ("el acusada es inocente") y si no hay realmente evidencia significativa para rechazar $H_{0}$, se prefiere "dejar libre al acusado" y evitar el error tipo I.

Así, en pruebas de hipótesis se controla el error tipo I por medio del nivel de significancia, que se recuerda seguidamente.

Definición 1.7 Nivel de significancia: es el valor máximo aceptable para $\alpha$, la probabilidad de que ocurra el error tipo I.

Por lo general, el nivel de significancia de una prueba es dado. Note que formalmente este valor no es la probabilidad $\alpha$ del error tipo I, sino una cota superior para $\alpha$. Sin embargo, si se considera $\alpha$ igual al nivel de significancia, entonces $\theta_{c}$ será el valor crítico permitido que genere el máximo error tipo I aceptable y por lo tanto el más cercano a $\theta_{0}$. Además, bajo esta consideración, el nivel de significancia es la medida de la región de rechazo.

Ejemplo 1.9 Considere la prueba

$$
H_{0}: \theta=\theta_{0} \quad(\geq) \quad H_{1}: \theta<\theta_{0}
$$

Denotemos $\operatorname{con} \delta$ el nivel de significancia y $\operatorname{con} \alpha$ la probabilidad del error tipo I. Se tiene que

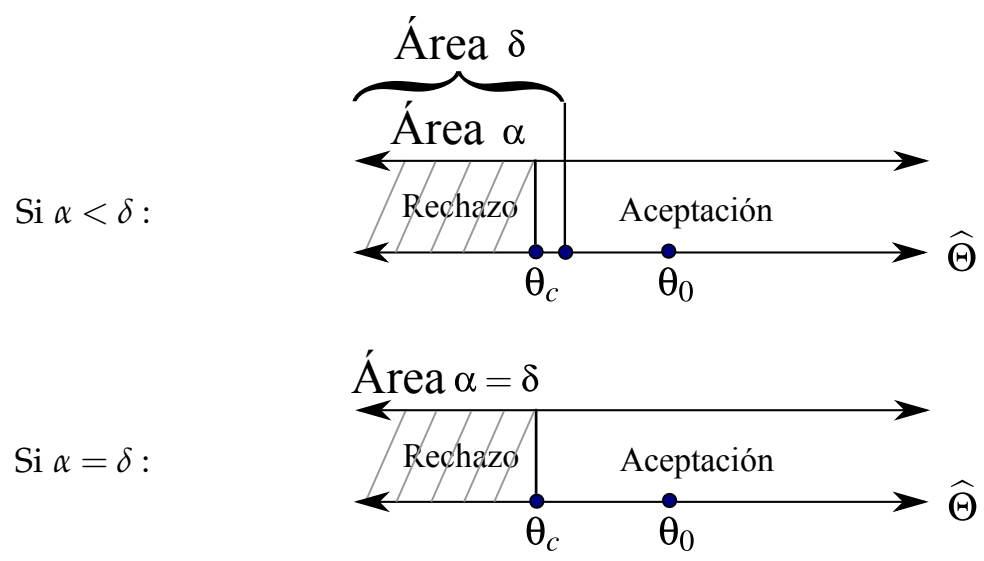

Note que entre más cerca esté $\theta_{\mathcal{c}}$ de $\theta_{0}$, como se comentó anteriormente, aumenta la probabilidad de cometer el error tipo I. Esto se debe a que se reduce la tolerancia para aceptar que $\theta=\theta_{0}$ a partir de estimaciones de $\theta$. Por otro lado, lo más cerca que puede estar $\theta_{\mathcal{c}}$ de $\theta_{0}$ es cuanto el error tipo I ha llegado a su máximo permitido, esto sucede si $\alpha=\delta$.

El nivel de significancia es escogido dependiendo de las exigencias con respecto al error tipo I. Como se ha mencionado, las pruebas de hipótesis que se estudiarán asumen un nivel de significancia y por lo tanto controlan el error tipo I. Por el contrario el error tipo II no puede controlarse en general y solo puede calcularse para valores específicos de $H_{1}$. 
Ejemplo 1.10 Considere la siguiente prueba de hipótesis

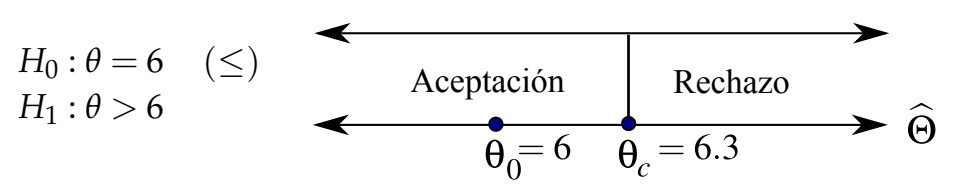

donde $\theta_{\mathcal{c}}=6.3$ y además $\widehat{\Theta} \sim N(\theta, 0.7)$. En este caso, la probabilidad del error tipo I es

$$
\begin{aligned}
\alpha & =P\left(H_{1} \mid H_{0}\right)=P(\widehat{\Theta}>6.3 \mid \theta=6)=P\left(\mathrm{Z}>\frac{6.3-6}{\sqrt{0.7}}\right) \\
& =P(\mathrm{Z}>0.358569) \approx 0.359424=35.9424 \% .
\end{aligned}
$$

Por otro lado, la probabilidad del error tipo II para la hipótesis alternativa específica $H_{1}^{\prime}: \theta=6.5$ es

$$
\begin{aligned}
\beta & =P\left(H_{0} \mid H_{1}^{\prime}\right)=P(\widehat{\Theta}<6.3 \mid \theta=6.5)=P\left(Z<\frac{6.3-6.5}{\sqrt{0.7}}\right) \\
& =P(Z<-0.239046) \approx 0.405165=40.5165 \%
\end{aligned}
$$

Como se mencionó los errores son en cierta medida opuestos. Dada que el valor contralado es $\alpha$, entonces sucede uno de los siguientes efectos: si se disminuye $\alpha$ entonces aumenta $\beta$, si se aumenta $\alpha$ entonces disminuye $\beta$. Sin embargo, se pueden disminuir ambos si se aumenta el tamaño de la muestra, de la cual se obtiene la estimación de $\theta$. Esto se verá más adelante para ciertas pruebas de hipótesis.

\subsection{Contraste de hipótesis}

¿Cómo realizar una prueba de hipótesis? Para realizar el contraste de hipótesis existen dos enfoques que se explican seguidamente.

\subsubsection{Determinación de regiones}

Este enfoque, llamado por algunos autores como enfoque clásico consiste en hallar el o los valores críticos a partir del nivel de significancia dado. El enfoque sigue los siguientes pasos

Paso 1. Redactar $H_{0}$ y $H_{1}$

Paso 2. Especificar el criterio de prueba o contraste:
a) Nivel de significancia $\alpha$
b) Distribución muestral de $\widehat{\Theta}$
c) Identificar las regiones de aceptación y rechazo tomando $\alpha$ como la probabilidad del error tipo I

Como se discutió antes, al tomar el nivel de significancia como la probabilidad el error tipo I, se está analizan el caso límite en que es más probable cometer el error de rechazar $H_{0}: \theta=\theta_{0}$ para estimaciones de $\theta$ cercanas a $\theta_{0}$.

Paso 3. Determinar el o los valores críticos, de acuerdo a el criterio de contraste (paso 2). Para ello, se redacta la probabilidad del error tipo I.

Paso 4. Datos muestrales: hallar un valor $\widehat{\theta}$ de $\widehat{\Theta}$ 
Paso 5. Decisión. Sea $R$ la región de rechazo

Si $\widehat{\theta} \in R$ entonces se rechaza $H_{0}$

Si $\widehat{\theta} \notin R$ entonces se acepta $H_{0}$

Paso 6. Conclusión

Si se rechaza $H_{0}$, se dice que si hay evidencia para rechazarla

Si se acepta $H_{0}$, se dice que no hay evidencia significativa en contra de $H_{0}$

Ejemplo 1.11 Dada una población se afirma que uno de sus parámetros $\theta$ no es menor a 4. Suponga que el estadístico $\widehat{\Theta}$ asociado a $\theta$ se distribuye normalmente

$$
\widehat{\Theta} \sim N(\theta, 0.25)
$$

para muestras de un tamaño $n$. Además, para una muestra de ese tamaño se observo un valor del estadístico de $\widehat{\theta}=3$. Realice el contraste de hipótesis utilizando el enfoque clásico con un nivel de significancia de 0.1.

1. Redacción de $H_{0}$ y $H_{1}$

Afirmación: $\theta \geq 4$

$$
H_{0}: \theta=4(\geq), \quad H_{1}: \theta<4
$$

\section{Criterio de contraste:}

a) Nivel de significancia $\alpha=0.1$

b) Distribución muestral de $\widehat{\Theta} \sim N$

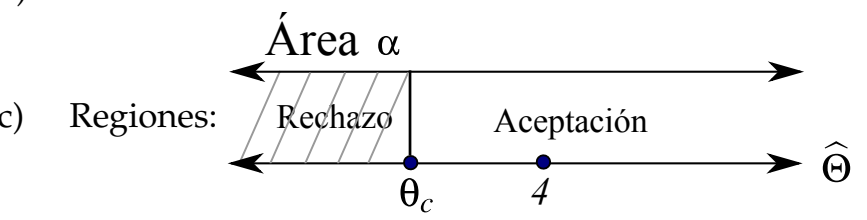

\section{Determinación del valor crítico}

$$
0.1=\alpha=P\left(H_{1} \mid H_{0}\right)=P\left(\widehat{\Theta}<\theta_{c} \mid \theta=4\right)=P\left(\mathrm{Z}<\frac{\theta_{c}-4}{\sqrt{0.25}}\right)
$$

Así

$$
P\left(Z<\frac{\theta_{c}-4}{\sqrt{0.25}}\right)=0.1 \Longrightarrow \frac{\theta_{c}-4}{\sqrt{0.25}}=-1.28 \Longrightarrow \theta_{c} \approx 3.36
$$

Por lo tanto las regiones son

$$
\text { Región de Aceptación: } A=] 3.36,+\infty[, \quad \text { Región de Rechazo: } R=]-\infty, 3.36[
$$

4. Datos muestrales: $\widehat{\theta}=3$.

5. Decisión. Como $\widehat{\theta}=3 \in R$ entonces se rechaza $H_{0}$.

6. Conclusión. Hay evidencia significativa para rechazar $H_{0}$. Dado que la afirmación se encuentra en $H_{0}$, entonces hay evidencia significativa para rechazar la afirmación. 


\subsubsection{Valor $\mathrm{P}$ de la prueba}

Definición 1.8 El valor $\mathbf{P}$ de una prueba de hipótesis es la probabilidad de que el estadístico $\widehat{\Theta}$ tenga el valor observado en la muestra o un valor extremo (en dirección de $H_{1}$ ) cuando $H_{0}$ es verdadera. Es decir es el nivel de significancia más bajo en el que el valor observado es significativo para rechazar $H_{0}$.

En otras palabras, el valor $P$, indica el valor mínimo de la posibilidad de que ocurra el error tipo I cuando el valor observado se considera parte de la región de rechazo. Note que el valor $P$ está relacionado con el nivel de significancia. La redacción del valor $P$ depende del tipo de prueba:

\begin{tabular}{|c|c|}
\hline Hipótesis & Valor $\mathbf{P}$ \\
\hline $\begin{array}{l}H_{0}: \theta=\theta_{0} \quad(\geq) \\
H_{1}: \theta<\theta_{0}\end{array}$ & $P\left(\widehat{\Theta} \leq \widehat{\theta} \mid H_{0}\right)$ \\
\hline $\begin{array}{l}H_{0}: \theta=\theta_{0} \quad(\leq) \\
H_{1}: \theta>\theta_{0}\end{array}$ & $P\left(\widehat{\Theta} \geq \widehat{\theta} \mid H_{0}\right)$ \\
\hline $\begin{array}{l}H_{0}: \theta=\theta_{0} \\
H_{1}: \theta \neq \theta_{0}\end{array}$ & $\begin{array}{l}\text { Sean } a \text { y } b \text { los valores dador por el valor observado } \widehat{\theta} \\
\text { y su simétrico respecto a } \theta_{0}: \\
\text { Valor } P=P\left(\widehat{\Theta}<a \mid H_{0}\right)+P\left(\widehat{\Theta}>b \mid H_{0}\right) \\
\text { Valí, si } \widehat{\Theta} \text { tiene una distribución simétrica respecto a } \theta_{0} \text { entonces: } \\
\text { Valor }=\left\{\begin{array}{lll}2 P\left(\widehat{\Theta}<\widehat{\theta} \mid H_{0}\right) & \text { si } & \widehat{\theta}<\theta_{0} \\
2 P\left(\widehat{\Theta}>\widehat{\theta} \mid H_{0}\right) & \text { si } & \widehat{\theta}>\theta_{0}\end{array}\right.\end{array}$ \\
\hline
\end{tabular}

Ejemplo 1.12 Se afirma que $\mu>5$ y se observa una muestra un valor de $\bar{x}=4.5$. Entonces se tiene que

$$
\begin{gathered}
\text { Afirmación: } \mu>5 \\
H_{0}: \mu=5(\leq), \quad H_{1}: \mu>5 \\
\text { Valor } P=P\left(\widehat{\Theta} \geq \widehat{\theta} \mid H_{0}\right)=P(\bar{X} \geq 4.5 \mid \mu=5)
\end{gathered}
$$

Para este enfoque se siguen los siguientes pasos

Paso 1. Redactar $H_{0}$ y $H_{1}$

Paso 2. Valor observado (datos muestrales): hallar un valor $\widehat{\theta}$ de $\widehat{\Theta}$

Paso 3. Redacción y determinación del valor $P$.

Paso 4. Decisión.

a) Si se conoce el nivel de significancia $\alpha$. 
a.1) Si el valor $P$ es menor o igual a $\alpha$. Aceptando $H_{0}$, se tiene que la probabilidad de que $\widehat{\Theta}$ tenga el valor observado en dirección a $H_{1}$ es menor que $\alpha$, el área de la región de rechazo, por ejemplo

$$
H_{0}: \theta=\theta_{0} \quad(\geq), \quad H_{1}: \theta<\theta_{0}
$$

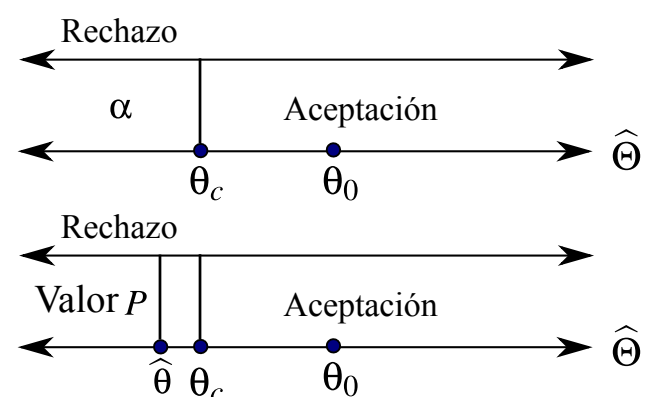

Por lo tanto, el valor observado está en la región de rechazo y se rechaza $H_{0}$.

a.2) Si el valor $P$ es mayor a $\alpha$. En este caso, por justificaciones similares, se acepta $H_{0}$.

En resumen:

Si el Valor $P \leq \alpha$ entonces se rechaza $H_{0}$

Si el Valor $P>\alpha$ entonces se acepta $H_{0}$

b) Si no se conoce el nivel de significancia $\alpha$. Se utiliza $\alpha=0.05$.

Paso 5. Conclusión

Si se rechaza $H_{0}$, se dice que si hay evidencia para rechazarla

Si se acepta $H_{0}$, se dice que no hay evidencia significativa a favor de $H_{1}$

Ejemplo 1.13 Dada una población se afirma que uno de sus parámetros $\theta$ no es menor a 4. Suponga que el estadístico $\widehat{\Theta}$ asociado a $\theta$ se distribuye normalmente

$$
\widehat{\Theta} \sim N(\theta, 0.25)
$$

para muestras de un tamaño $n$. Además para una muestra de ese tamaño se observó un valor del estadístico de $\widehat{\theta}=3$. Realice el contraste de hipótesis utilizando Valor $P$ con un nivel de significancia de 0.1 .

1. Redacción de $H_{0}$ y $H_{1}$

$$
\begin{gathered}
\text { Afirmación: } \theta \geq 4 \\
H_{0}: \theta=4(\geq), \quad H_{1}: \theta<4
\end{gathered}
$$

2. Valor observado: $\widehat{\theta}=3$.

3. Valor $P$ :

$$
\text { Valor } P=P(\widehat{\Theta} \leq 3 \mid \theta=4)=P\left(Z \leq \frac{3-4}{\sqrt{0.25}}\right)=P(Z \leq-2)=0.022750
$$

4. Decisión. Como Valor $P<0.1=\alpha$ entonces se rechaza $H_{0}$.

5. Conclusión. Hay evidencia para rechazar $H_{0}$, y como la afirmación se encuentra en $H_{0}$, se rechaza la afirmación. 


\subsubsection{Cuál de los dos enfoques utilizar?}

Ambos enfoques son equivalentes; sin embargo, al compararlos se tiene que

\begin{tabular}{|c|c|c|}
\hline Enfoque & $\begin{array}{c}\text { Utilización de valor } \\
\text { muestral } \widehat{\theta}\end{array}$ & $\begin{array}{c}\text { Utilización del nivel } \\
\text { de significancia } \alpha\end{array}$ \\
\hline Determinación de regiones & $\begin{array}{c}\text { Al final, para ver en qué } \\
\text { región se encuentra } \widehat{\theta}\end{array}$ & $\begin{array}{c}\text { Al inicio, para calcular } \\
\text { las regiones }\end{array}$ \\
\hline Valor $P$ & $\begin{array}{c}\text { Al inicio, para calcular } \\
\text { el Valor } P\end{array}$ & $\begin{array}{c}\text { Al final, al comparar } \\
\alpha \text { y el Valor } P\end{array}$ \\
\hline
\end{tabular}

Así se tiene que

1. Se utiliza el valor $P$ cuando se conoce el valor muestral y no se tiene certeza sobre cuál nivel de significancia utilizar. Por ejemplo, si el valor $P$ es 0.007 , indica que se puede utilizar un nivel de significancia bastante pequeño para rechazar la hipótesis como 0.01 en lugar de utilizar un nivel de significancia a priori del $10 \%$ por ejemplo. Note que al reportar un valor pequeño de $\alpha$ en una prueba que se rechaza, la conclusión tiene una mejor aceptación. Particularmente el valor $P$ es útil cuando se tiene un valor muestral difícil de volver a conseguir, ya sea por razones económicas o de tiempo y se quiere manipular el nivel de significancia.

2. Se utiliza la determinación de regiones cuando aún no se tiene el valor muestral y se tiene certeza del nivel de significancia a utilizar. Así, con el nivel de confianza se pueden determinar las regiones de prueba y luego tomar la muestra para obtener un valor muestral y ubicarlo en alguna de las regiones. Se puede dar un efecto negativo y no ético de la estadística, que es la manipulación del valor muestral para ajustarlo a conveniencia.

Para fines prácticos de este documento, se utiliza el valor $P$ cuando el ejercicio no indique el nivel de significancia a utilizar. En caso contrario, el lector puede escoger el enfoque a utilizar.

\subsection{Algunos ejemplos}

Considere la población dada por la variable aleatoria $X$ que con media poblacional $\mu$ y varianza poblacional $\sigma^{2}$. En las pruebas de un promedio la hipótesis nula es de la forma

$$
H_{0}: \mu=\mu_{0}
$$

Si $\bar{X}$ sigue una distribución normal para muestras de tamaño $n$ (la población $X$ es normal o $n \geq 30$ ) y se conoce $\sigma$ entonces

$$
\bar{X} \sim N\left(\mu, \sigma^{2}\right) \quad \Longrightarrow \quad Z=\frac{\bar{X}-\mu}{\sigma / \sqrt{n}} \sim N(0,1)
$$

y bajo la hipótesis $H_{0}: \mu=\mu_{0}$, se tiene que

$$
\mathrm{Z}=\frac{\bar{X}-\mu_{0}}{\sigma / \sqrt{n}}
$$

sigue una distribución normal estándar. ${ }^{1}$.

Ejemplo 1.14 (¿La directiva tiene razón?). El sindicato de empleados de una empresa ubicada en una zona rural ha solicitado que la hora de entrada sea a las 7:15am y no a las 7:00 am, esto debido a que la mayoría de los empleados no viven cerca de la empresa. La directiva ha denegado la solicitud pues considera que el empleado

\footnotetext{
${ }^{1}$ En realidad, si $X \sim N$ entonces $\bar{X}$ sigue una distribución normal. Pero si $n \geq 30$ entonces $\bar{X}$ sigue una distribución que se aproxima a la normal, esto se denota $\bar{X} \underset{\text { aprox }}{\sim} N$.
} 
promedio no recorre más de 9 kilómetros diariamente para llegar a la empresa. Se desea contrastar la afirmación emitida por dicha directiva con un nivel de significancia de 0.05. Para ello, en una muestra de 50 empleados se observó un recorrido promedio de $9.1 \mathrm{~km}$ con una desviación estándar de $5 \mathrm{~km}$.

1. Plantee la hipótesis nula, la hipótesis alternativa y las regiones de aceptación y rechazo.

Sea $\mu$ la distancia promedio, en kilómetros, que recorre diariamente el empleado para llegar a la empresa.

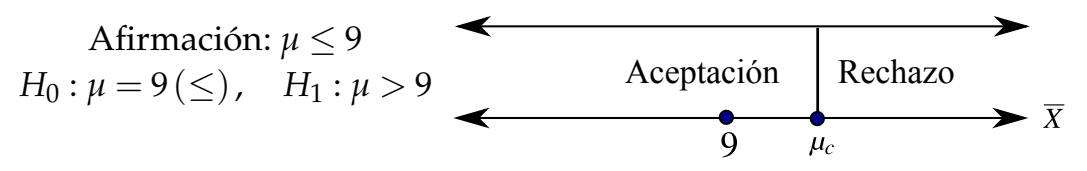

2. Determine las regiones de aceptación y rechazo.

Dado que el tamaño de muestra es $n=50$ entonces

$$
\bar{X} \underset{\text { aprox }}{\sim} N \quad y \quad \sigma \approx s=5
$$

Además $\alpha=0.05$, por lo tanto

$$
\begin{array}{ccc} 
& \alpha=P\left(H_{1} \mid H_{0}\right) \\
& & 0.05=P\left(\bar{X} \geq \mu_{c} \mid \mu=9\right)=P\left(Z>\frac{\mu_{c}-9}{5 / \sqrt{50}}\right) \\
\Longrightarrow & \frac{\mu_{c}-9}{5 / \sqrt{50}}=z_{0.95}=1.645 \\
& & \mu_{c}=10.167
\end{array}
$$

Región de rechazo: $R=] 10.1632,+\infty[\quad y \quad$ Región de aceptación: $A=]-\infty, 10.1632[$.

3. ¿Hay evidencia en contra de la afirmación de la directiva?

El valor observado es $\bar{x}=9.1$, como $\bar{x} \in A$ entonces se acepta $H_{0}$. Dado que la afirmación de la directiva fue ubicada en $H_{0}$, entonces se acepta la afirmación, por lo tanto no se encontró evidencia significativa en contra de lo expresado por la directiva.

Ejemplo 1.15 (¿El sindicato tiene razón?). Considere el ejemplo anterior. Ante lo expresado por la directiva, el sidicato señala que el empleado promedio recorre al menos $9.2 \mathrm{~km}$ para llegar a la empresa. Utilizando los mismos datos del ejemplo anterior, ¿aceptaría la afirmación del sindicato?

Note que la afirmación de la directiva $(\mu \leq 9)$ es opuesta a la afirmación del sidicato $(\mu \geq 9.2)$. Como la afirmación de la directiva se considera aceptable (ejemplo anterior), se puede cometer el error de rechazar la afirmación del sindicato. En pruebas de hipótesis, cada afirmación debe ser analizada por separado. Analicemos la afirmación del sindicato

Afirmación: $\mu \geq 9.2$

$$
H_{0}: \mu=9.2(\geq), \quad H_{1}: \mu<9.2
$$

Como $n=50$ entonces $\bar{X} \underset{\text { aprox }}{\sim} N$ y $\sigma \approx s=5$. Entonces el valor $P$ de la prueba es

$$
\text { Valor } \begin{aligned}
P & =P\left(\bar{X} \leq \bar{x} \mid H_{0}\right)=P(\bar{X} \leq 9.1 \mid \mu=9.2) \\
& =P\left(Z \leq \frac{9.1-9.2}{5 / \sqrt{50}}\right)=P(Z \leq-0.141421) \\
& =0.444330
\end{aligned}
$$

Dado que el Valor $P>0.05$, se acepta $H_{0}$ y entonces se acepta la afirmación. Por lo tanto, no hay evidencia significativa para rechazar la afirmación del sindicato 
Ejemplo 1.16 (¿La directiva o el sindicato?). En los ejemplos anteriores, se aceptan las afirmaciones de la directiva de la empresa y del sindicato, pero estas afirmaciones son contradictorias, ¿qué está sucediendo?

Lo que sucede es que la información muestral es insificiente para rechazar alguna de las afirmaciones. Además se debe recordar que el concepto de aceptación en pruebas de hipótesis es distinto al usado comúnmente, ambas afirmaciones se aceptan porque no hay evidencias significativas en contra. Aquí se recomienda hacer el estudio con una nueva muestra.

\section{Bibliografía}

[1] Acuña, Luis. Estadística Aplicada con Fathom. Editorial Tecnológica de Costa Rica: Cartago, Costa Rica. 2004.

[2] Aliaga, Martha. Estadística Interactiva. Encuentro Latinoamericano de Educación Estadística (ELEE). 4 y 5 de Julio de 2008 en el Instituto Tecnológico y de Estudios Superiores de Monterrey, México. 2008.

[3] Antibí, André. Didáctica de las Matemáticas: Métodos de Resolución de problemas. Editorial Serie Cabécar: San José, Costa Rica. 2000.

[4] Devore, Jay. Probabilidad y Estádistica para Ingeniería y Ciencias, Cuarta edición. International Thomson Editores: México. 1998.

[5] Gómez, Miguel. Elementos de estadísitca descriptiva. EUNED: San José, Costa Rica. 2000.

[6] Mora, Eric. Curso Intermedio de Probabilidades. Editorial de la Universidad de Costa Rica: San José, Costa Rica. 2010.

[7] Sanabria, Giovanni. Tópicos precedentes al estudio de la Teoría de Probabilidades, II semestre del 2007. Publicaciones ITCR. 2007.

[8] Sanabria, Giovanni. Una propuesta para la enseñanza de los Elementos de Análisis Combinatorio. Memorias del Primer Encuentro Nacional en la Enseñanza de la Probabilidad y la Estadística ( $1^{\circ}$ ENEPE), Puebla - México del 16 al 18 de junio del 2010.

[9] Sanabria, Giovanni. Comprendiendo la Estadística Inferencial. Editorial Tecnológica de Costa Rica: Cartago, Costa Rica. 2011.

[10] Walpole, Ronald; Myers, Raymond; Myers, Sharon. Probabilidad y estadistica para ingenieros, Sexta edición. PrenticeHall Hispanoamericana. S.A: México. 1999.

[11] Vallecillos, A., y Batanero, C. Análisis del aprendizaje de conceptos clave en el contraste de hipótesis estadísticas mediante el estudio de casos. Recherches en Didactique des Mathématiques, 17(1), 29-48. 1997.

Una propuesta para introducir la enseñanza de las Pruebas de Hipótesis. Giovanni Sanabria B.

Derechos Reservados ( 2012 Revista digital Matemática, Educación e Internet (www.tec-digital.itcr.ac.cr/revistamatematica/) 